\title{
Spatial-Temporal Synchronous Graph Convolutional Networks: A New Framework for Spatial-Temporal Network Data Forecasting
}

\author{
Chao Song, ${ }^{1,2}$ Youfang Lin, ${ }^{1,2,3}$ Shengnan Guo, ${ }^{1,2}$ Huaiyu Wan ${ }^{1,2,3 *}$ \\ ${ }^{1}$ School of Computer and Information Technology, Beijing Jiaotong University, Beijing, China \\ ${ }^{2}$ Beijing Key Laboratory of Traffic Data Analysis and Mining, Beijing, China \\ ${ }^{3}$ CAAC Key Laboratory of Intelligent Passenger Service of Civil Aviation, Beijing, China \\ \{chaosong, yflin, guoshn, hywan\}@bjtu.edu.cn
}

\begin{abstract}
Spatial-temporal network data forecasting is of great importance in a huge amount of applications for traffic management and urban planning. However, the underlying complex spatial-temporal correlations and heterogeneities make this problem challenging. Existing methods usually use separate components to capture spatial and temporal correlations and ignore the heterogeneities in spatial-temporal data. In this paper, we propose a novel model, named Spatial-Temporal Synchronous Graph Convolutional Networks (STSGCN), for spatial-temporal network data forecasting. The model is able to effectively capture the complex localized spatial-temporal correlations through an elaborately designed spatial-temporal synchronous modeling mechanism. Meanwhile, multiple modules for different time periods are designed in the model to effectively capture the heterogeneities in localized spatialtemporal graphs. Extensive experiments are conducted on four real-world datasets, which demonstrates that our method achieves the state-of-the-art performance and consistently outperforms other baselines.
\end{abstract}

\section{Introduction}

Spatial-temporal network data forecasting is a fundamental research problem in spatial-temporal data mining. The spatial-temporal network is a typical data structure that can describe lots of data in many real-world applications, such as traffic networks, mobile base-station networks, urban water systems, etc. Accurate predictions of spatial-temporal network data can significantly improve the service quality of these applications. With the development of deep learning on graphs, powerful methods like graph convolutional networks and its variants have been widely applied to these spatial-temporal network data prediction tasks and achieved promising performance. However, there is still a lack of effective methods to model the correlations and heterogeneity in both the spatial and temporal aspects. In this paper, we focus on designing a model to synchronously capture the complex spatial-temporal correlations and take the heterogeneity into account to improve the accuracy of spatial-temporal network data forecasting.

\footnotetext{
${ }^{*}$ Corresponding author: hywan@ bjtu.edu.cn Copyright (c) 2020, Association for the Advancement of Artificial Intelligence (www.aaai.org). All rights reserved.
}

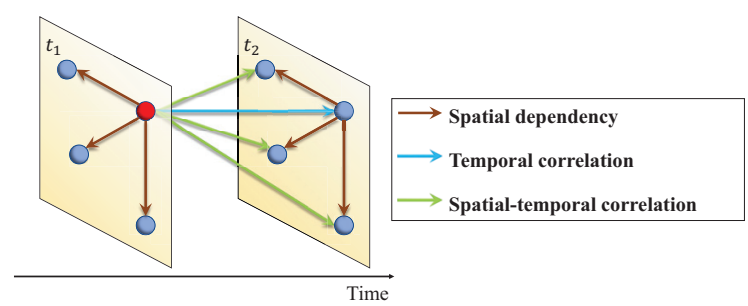

Figure 1: The influence of the red node in a spatial-temporal network. The brown arrows denote the edges of the network, and also indicate the influence in the spatial dimension. The blue arrow indicates the influence of the red node on itself at the next time step. The green arrows indicate the influence that across both the spatial and temporal dimensions, between the red node and its neighbors at the next time step. $t_{1}$ and $t_{2}$ denotes two continuous time steps.

Taking the spatial-temporal network shown in Figure 1 as an example, there are three different influences in this network. Each node in the spatial-temporal graph can directly influence its neighbor nodes at the same time step, and such influence is derived from the actual spatial dependencies. Meanwhile, each node can also directly influence itself at the next time step due to the temporal correlations in time series. Besides, each node can even directly influence its neighbor nodes at the next time step because of the synchronous spatial-temporal correlations, as shown in Figure 1. The reason for the existence of the three different kinds of influences is that the information propagation in a spatialtemporal network occurs both along the spatial and temporal dimensions simultaneously. Due to the restriction of the spatial distances between nodes and the time range of the time series, these complex spatial-temporal correlations are usually localized. We refer to these complex influences as localized spatial-temporal correlations. Modeling such correlations is crucial for spatial-temporal network data prediction. Previous studies like DCRNN (Li et al. 2017), STGCN (Yu, Yin, and Zhu 2018) and ASTGCN (Guo et al. 2019a) use two separate components to capture temporal and spatial dependencies, respectively. These methods only directly capture the first two kinds of influences we mentioned above, 
namely the spatial dependencies and the temporal correlations. They feed the spatial representations into the temporal modeling modules to capture the third kind of influence indirectly. However, we believe that if these complex localized spatial-temporal correlations can be captured simultaneously, it will be very effective for spatial-temporal data prediction because this modeling method exposes the fundamental way how spatial-temporal network data are generated.

Besides, spatial-temporal network data usually exhibit heterogeneity in both the spatial and temporal dimensions. For example, in a citywide road network, the observations recorded by traffic monitoring stations in residential and commercial areas tend to exhibit different patterns at different times. However, many previous studies use shared modules for different time periods, which cannot effectively capture the heterogeneity in spatial-temporal networks.

To capture the complex localized spatial-temporal correlations and the heterogeneity in spatial-temporal data, we propose a model called Spatial-Temporal Synchronous Graph Convolutional Network (STSGCN). Different from many previous works, the STSGCN model can simultaneously capture the localized spatial-temporal correlations directly, instead of using different types of deep neural networks to model the spatial dependencies and temporal correlations separately. Specifically, we construct localized spatial-temporal graphs which connect individual spatial graphs of adjacent time steps into one graph. Then we construct a Spatial-Temporal Synchronous Graph Convolutional Module (STSGCM) to capture the complex localized spatial-temporal correlations in these localized spatialtemporal graphs. Meanwhile, to capture the heterogeneity in long-range spatial-temporal network data, we design a Spatial-Temporal Synchronous Graph Convolutional Layer (STSGCL), which deploys multiple individual STSGCMs on different time periods. Finally, we stack multiple STSGCLs to aggregate long-range spatial-temporal correlations and heterogeneity for prediction.

Overall, the contributions of our work are as follows:

- We propose a novel spatial-temporal graph convolutional module to synchronously capture the localized spatialtemporal correlations directly, instead of using different types of neural network modules separately.

- We construct a multi-module layer to capture the heterogeneity in long-range spatial-temporal graphs. This multimodule layer deploys multiple modules on each time period, allowing each module to focus on extracting spatialtemporal correlations on each localized spatial-temporal graph.

- Extensive experiments are conducted on four real-world datasets and the experimental results show that our model consistently outperforms all the baseline methods.

\section{Related Work \\ Spatial-Temporal Prediction}

The spatial-temporal data prediction problem is a very important research topic in spatial-temporal data mining. Many of classic methods like ARIMA (Williams and Hoel 2003) and SVM (Drucker et al. 1997) only take temporal information into account. It is challenging to integrate complex spatial dependencies into prediction methods. The ConvLSTM (Shi et al. 2015) model is an extension of fully-connected LSTM (Graves 2013), which combines CNN and RNN to model spatial and temporal correlations respectively. It utilizes CNN's powerful capability in spatial information extraction. ST-ResNet (Zhang, Zheng, and Qi 2017) is a CNN based deep residual network for citywide crowd flows prediction, which shows the power of deep residual CNN on modeling spatial-temporal grid data. ST-3DNet (Guo et al. 2019b) introduces 3D convolutions into this area, which can effectively extract features from both the spatial and temporal dimensions. It uses two components to model the local temporal patterns and the long-term temporal patterns respectively. All of these methods above are designed for spatial-temporal grid data.

Recently, researchers try to utilize graph convolution methods to model the spatial correlations in spatial-temporal network data. DCRNN (Li et al. 2017) introduces graph convolutional networks into spatial-temporal network data prediction, which employs a diffusion graph convolution network to describe the information diffusion process in spatial networks. It uses RNN to model temporal correlations like ConvLSTM. STGCN (Yu, Yin, and Zhu 2018) uses CNN to model temporal correlations. ASTGCN (Guo et al. 2019a) uses two attention layers to capture the dynamics of spatial dependencies and temporal correlations. Graph WaveNet (Wu et al. 2019) designs a self-adaptive matrix to take the variations of the influence between nodes and their neighbors into account. It uses dilated casual convolutions to model the temporal correlations to increase the receptive field exponentially.

However, all of the above methods used two different components to capture spatial dependencies and temporal correlations separately. Differ from them, STG2Seq (Bai et al. 2019) tries to model spatial-temporal correlations simultaneously by using a gated residual GCN module with two attention mechanisms. However, to some extent, concatenating features of each node in different time steps obscures spatial-temporal correlations. And it cannot capture the heterogeneity in spatial-temporal data.

\section{Graph Convolution Network}

Graph convolutional network (GCN) has achieved extraordinary performance on several different types of tasks based on the graph structure, such as node classification and network representation. Spectral GCNs are defined in the spectral domain. Lots of methods are derived from the work of (Bruna et al. 2013). ChebNet (Defferrard, Bresson, and Vandergheynst 2016) is a powerful GCN that utilizes the Chebyshev extension to reduce the complexity of laplacians computation. GCN (Kipf and Welling 2017) simplifies ChebNet to a more simple form and achieves state-of-the-art performance on various tasks. Spatial GCN generalizes the traditional convolutional network from the Euclidean space to the vertice domain. GraphSAGE (Hamilton, Ying, and Leskovec 2017) samples a fixed number of neighbors for 
each node in the graph and aggregates the features of their neighbors and themselves. GAT (Veličković et al. 2018) is a powerful GCN variant defined in the vertice domain, which uses attention layers to adjust the importance of neighbor nodes dynamically.

\section{Preliminaries}

- Definition 1: Spatial network $\mathcal{G}$. We use $\mathcal{G}=(V, E, A)$ to denotes a spatial network, where $|V|=N$ is the set of vertices, $N$ denotes the number of vertices, and $E$ denotes the set of edges. $A$ is the adjacency matrix of network $\mathcal{G}$. The spatial network $\mathcal{G}$ represents the relationship between the nodes in the spatial dimension, and the network structure does not change with time. In our work, this spatial network can be either directed or undirected.

- Definition 2: Graph signal matrix $X_{\mathcal{G}}^{(t)} \in \mathbb{R}^{N \times C}$, where $C$ is the number of attribute features, $t$ denotes the time step. This graph signal matrix represents the observations of the spatial network $\mathcal{G}$ at the time step $t$.

The problem of spatial-temporal network data forecasting can be described as: learning a mapping function $f$ which maps the historical spatial-temporal network series $\left(X_{\mathcal{G}}^{(t-T+1)}, X_{\mathcal{G}}^{(t-T+2)}, \ldots, X_{\mathcal{G}}^{(t)}\right)$ into the future observations of this spatial-temporal network $\left(X_{\mathcal{G}}^{(t+1)}, X_{\mathcal{G}}^{(t+2)}, \ldots, X_{\mathcal{G}}^{\left(t+T^{\prime}\right)}\right)$, where $T$ denotes the length of historical spatial-temporal network series, $T^{\prime}$ denotes the length of the target spatial-temporal network series to forecast.

\section{Spatial-Temporal Synchronous Graph Convolutional Network}

Figure 2 illustrates the architecture of our STSGCN model. We summarize the core idea of STSGCN as three points: 1) Connect each node with itself at the previous and the next time steps to construct a localized spatial-temporal graph. 2) Use a Spatial-Temporal Synchronous Graph Convolutional Module to capture the localized spatial-temporal correlations. 3) Deploy multiple modules to model heterogeneities in spatial-temporal network series.

\section{Localized Spatial-Temporal Graph Construction}

We intend to build a model that can directly capture the impact of each node on its neighbors that belongs to both the current and the adjacent time steps. The most intuitive idea to achieve this goal is to connect all nodes with themselves at the adjacent time steps (Figure 3 (a)). By connecting all nodes with themselves at the previous and the next moments, we can get a localized spatial-temporal graph. According to the topological structure of the localized spatial-temporal graph, the correlations between each node and its spatialtemporal neighbors can be captured directly.

We use $A \in \mathbb{R}^{N \times N}$ to denote the adjacency matrix of the spatial graph. $A^{\prime} \in \mathbb{R}^{3 N \times 3 N}$ denotes the adjacency matrix of the localized spatial-temporal graph constructed on three continuous spatial graphs. For node $i$ in the spatial graph, we can calculate its new index in the localized spatial-temporal

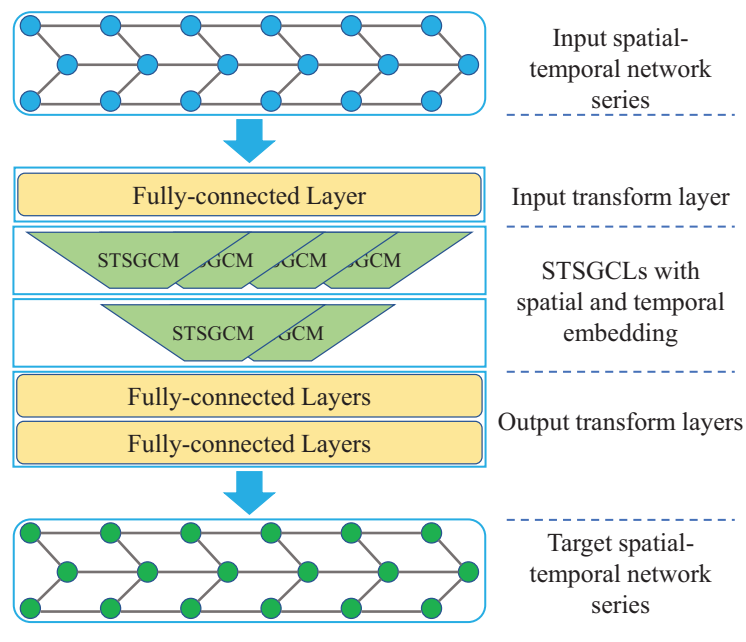

Figure 2: STSGCN architecture. Our STSGCN consists of multiple Spatial-Temporal Synchronous Graph Convolutional Layers (STSGCLs) with an input and an output layer. It uses an input layer to transform the input features into a higher dimensional space. Then stacked mulitple STSGCLs capture the localized spatial-temporal correlations and heterogeneities in spatial-temporal network series. Finally, it uses a multi-module output layer to map the final representations into the output space.

graph by $(t-1) N+i$, where $t(0<t \leq 3)$ denotes the time step number in the localized spatial-temporal graph. If two nodes connect with each other in this localized spatialtemporal graph, the corresponding value in the adjacency matrix is set to be 1 . The adjacency matrix of the localized spatial-temporal graph can be formulated as:

$$
A_{i, j}^{\prime}=\left\{\begin{array}{ll}
1, & \text { if } v_{i} \text { connects to } v_{j} \\
0, & \text { otherwise }
\end{array},\right.
$$

where $v_{i}$ denotes the node $i$ in localized spatial-temporal graph. The adjacency matrix $A^{\prime}$ contains $3 N$ nodes. Figure 3 (b) illustrates the adjacency matrix of the localized spatialtemporal graph. The diagonal of the adjacency matrix are the adjacency matrices of the spatial networks of three continuous time steps. The two sides of the diagonal indicate the connectivity of each node to itself that belongs to the adjacent time steps.

\section{Spatial-Temporal Embedding}

However, connecting the nodes at different time step into one graph obscures the time attribute of each node. In other words, this localized spatial-temporal graph puts the nodes at different time steps into a same environment without distinguishing them. Inspired by the ConvS2S(Gehring et al. 2017), we equip position embedding to the spatial-temporal network series so that the model can take the spatial and temporal information into account, which can enhance the ability to model the spatial-temporal correlations. For the spatial-temporal network series $X_{\mathcal{G}} \in \mathbb{R}^{N \times C \times T}$, we create a learnable temporal embedding matrix $T_{e m b} \in \mathbb{R}^{C \times T}$ 


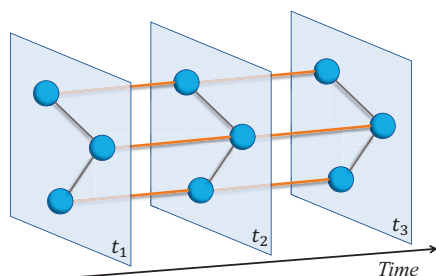

(a) Localized Spatial-Temporal Graph

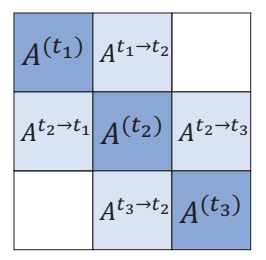

(b) Adjacency matrix of Localized Spatial-Temporal Graph
Figure 3: Localized Spatial-Temporal Graph construction. (a) is an example of a localized spatial-temporal graph. (b) is the adjacency matrix of the localized spatial-temporal graph in (a). $A^{\left(t_{i}\right)}$ denotes the adjacency matrix of the spatial graph at time step $i . A^{t_{i} \rightarrow t_{j}}$ denotes the connections between the nodes with themselves at the time step $i$ and $j$.

and a learnable spatial embedding matrix $S_{e m b} \in \mathbb{R}^{N \times C}$. After the training process is completed, the two embedding matrices will contains the necessary temporal and spatial information to help the model capture the spatial-temporal correlations.

We add these two embedding matrix to the spatialtemporal network series with broadcast operation to obtain the new representations of the network series:

$$
X_{\mathcal{G}+t_{e m b}+s_{\text {emb }}}=X_{\mathcal{G}}+T_{\text {emb }}+S_{\text {emb }} \in \mathbb{R}^{N \times C \times T} .
$$

\section{Spatial-Temporal Synchronous Graph Convolutional Module}

We build a Spatial-Temporal Synchronous Graph Convolutional Module (STSGCM) to capture localized spatialtemporal correlations. The STSGCM consists of a group of graph convolutional operations. Graph convolutional operations can aggregate the features of each node with its neighbors. We define a graph convolutional operation in the vertice domain to aggregate localized spatial-temporal features in spatial-temporal networks. The input of the graph convolutional operation is the graph signal matrix of the localized spatial-temporal graph. In our graph convolutional operation, each node aggregates the features of its own and its neighbors at adjacent time steps. The aggregate function is a linear combination whose weights are equal to the weights of the edges between the node and its neighbors. Then we deploy a fully-connected layer with an activation function to transform the features of nodes into a new space. This graph convolutional operation can be formulated as follow:

$$
G C N\left(h^{(l-1)}\right)=h^{(l)}=\sigma\left(A^{\prime} h^{(l-1)} W+b\right) \in \mathbb{R}^{3 N \times C^{\prime}},
$$

where $A^{\prime} \in \mathbb{R}^{3 N \times 3 N}$ denotes the adjacency matrix of the localized spatial-temporal graph, $h^{(l-1)} \in \mathbb{R}^{3 N \times C}$ is the input of the $l$-th graph convolutional layer, $W \in \mathbb{R}^{C \times C^{\prime}}$ and $b \in \mathbb{R}^{C^{\prime}}$ are learnable parameters, $\sigma$ denotes the activation function, such as ReLU and GLU (Dauphin et al. 2017). If we select GLU as the activation function of the graph convolutional layer, the graph convolutional layer can be described as follow:

$$
h^{(l)}=\left(A^{\prime} h^{(l-1)} W_{1}+b_{1}\right) \otimes \operatorname{sigmoid}\left(A^{\prime} h^{(l-1)} W_{2}+b_{2}\right),
$$

where $W_{1} \in \mathbb{R}^{C \times C^{\prime}}, W_{2} \in \mathbb{R}^{C \times C^{\prime}}, b_{1} \in \mathbb{R}^{C^{\prime}}, b_{2} \in \mathbb{R}^{C^{\prime}}$ are learnable parameters, sigmoid denotes the sigmoid activation function, i.e., $\operatorname{sigmoid}(x)=\frac{1}{1+e^{-x}}, \otimes$ denotes element-wise product. The gated linear unit controls which node's information can be passed to the next layer.

This graph convolutional operation is defined in the vertice domain, which means that it does not need to compute the graph laplacian. Also, this graph convolutional operation can be applied not only to undirected graphs but also to directed graphs. In addition, we add self-loop to each node in the localized spatial-temporal graph, in order to allow the graph convolutional operation to take its own characteristics into account when aggregating features.

We stack multiple graph convolutional operations to expand the aggregation area, which can increase the receptive field of the graph convolution operations to capture localized spatial-temporal correlations (Figure 4 (a)). We select JK-net (Xu et al. 2018) as the base structure of our STSGCM and design a new aggregation layer to filter useless information (Figure 4 (b), 4 (c)).

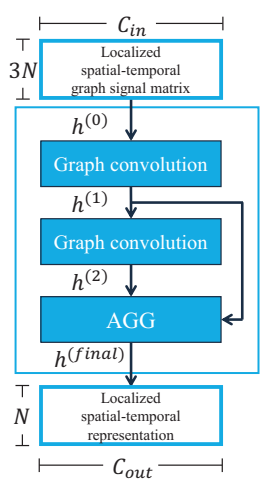

(a) STSGCM

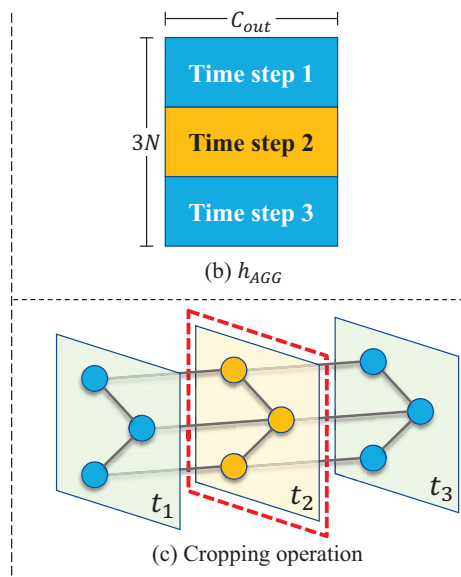

(c) Cropping operation
Figure 4: (a) is an example of the architecture of the SpatialTemporal Synchronous Graph Convolutional Module with two graph convolutional operations. $C_{i n}$ and $C_{\text {out }}$ denotes the number of features of the input matrix and the output matrix respectively, AGG denotes the aggregation layer. (b) denotes the output of the aggregating operation. (c) is an example of cropping operation in the aggregation layer, which only retain the nodes at the middle time step.

We use $h^{(l)}$ to denote the output of the $l$-th graph convolutional operation, where $h^{(0)}$ denotes the input of the first graph convolutional operation. For STSGCM with $L$ graph convolutional operations, the output of each graph convolution operation will be fed into an aggregation layer (Figure 4 (a)). The aggregation layer will compact the outputs of all 
layers in the STSGCM. The aggregation operation has two steps: aggregating and cropping.

Aggregating operation We select max-pooling as the aggregation operation. It applies an element-wise max operation to the outputs of all the graph convolutions in STSGCM. The max operation needs all outputs have the same size, so the number of the kernels for the graph convolutional operations within a module should be equal. The max aggregating operation can be formulated as:

$$
h_{A G G}=\max \left(h^{(1)}, h^{(2)}, \ldots, h^{(L)}\right) \in \mathbb{R}^{3 N \times C_{\text {out }}},
$$

where $C_{\text {out }}$ denotes the number of kernels in the graph convolutional operations.

Cropping operation The cropping operation (Figure 4 (c)) removes all the features of the nodes at the previous and the next time steps, and only the nodes in the middle moment are retained. The reason for that is the graph convolutional operations has already aggregated the information from the previous and the next time steps. Each node contains the localized spatial-temporal correlations even though we crop the two time steps. If we stack multiple STSGCMs and retain the features of all the adjacent time steps, much redundant information will reside in the model, which can seriously impair the performance of the model.

To sum up, the input to STSGCM is a localized spatialtemporal graph signal matrix $h^{(0)} \in \mathbb{R}^{3 N \times C_{i n}}$. After several graph convolutional operations, the outputs of each graph

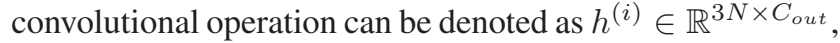
where $i$ denotes the operation index. The aggregating oper-

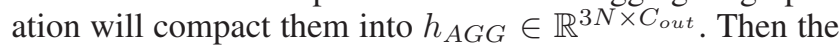
cropping operation retain the nodes at the middle time step, generate the output of STSGCM $h^{(\text {final })} \in \mathbb{R}^{N \times C_{\text {out }}}$. The green arrows in Figure 1 indicate the spaital-temporal correlations between the node and its two-hop neighbors in localized spatial-temporal graphs. The STSGCMs with at least two stacked graph convolutional operations can model the three different types of correlations indicated in Figure 1 directly.

\section{Spatial-Temporal Synchronous Graph Convolutional Layer}

To capture long-range spatial-temporal correlations of the entire network series, we use a sliding window to cut out different periods. Due to the heterogeneity in the spatialtemporal data, it is better to use multiple STSGCMs to model different periods rather than to share one for all periods. Multiple STSGCMs allow each one to focus on modeling the localized spatial-temporal correlations in the localized graph. We deploy a group of STSGCMs as a SpatialTemporal Synchronous Graph Convolutional Layer (STSGCL) to extract long-range spatial-temporal features, as shown in Figure 2.

We denote the input matrix of a STSGCL as $X \in$ $\mathbb{R}^{T \times N \times C}$. We add spatial-temporal embeddings for each STSGCL at first. Then the sliding window in the STSGCL will cuts out the input into $T-2$ spatial-temporal network series. Each spatial-temporal network series can be denoted as $X^{\prime} \in \mathbb{R}^{3 \times N \times C}$. We reshape them as $X_{\text {reshape }}^{\prime} \in \mathbb{R}^{3 N \times C}$, which can be fed into STSGCM with the localized spatialtemporal graph directly. STSGCL deploys $T-2$ STSGCMs on $T-2$ localized spatial-temporal graphs to capture the localized spatial-temporal correlations in these $T-2$ spatialtemporal network series. After that, all these $T-2$ STSGCMs' outputs are concatenated into one matrix as the output of STSGCL. That can be formulated as:

$$
M=\left[M_{1}, M_{2}, \ldots, M_{T-2}\right] \in \mathbb{R}^{(T-2) \times N \times C_{\text {out }}},
$$

where $M_{i} \in \mathbb{R}^{N \times C_{\text {out }}}$ denotes the outputs of the $i$-th STSGCM.

By stacking multiple STSGCLs, we can build a hierarchical model that can capture complex spatial-temporal correlations and spatial-temporal heterogeneity. After several spatial-temporal synchronous graph convolution operations, each node will contain the localized spatial-temporal correlations centered by itself.

\section{Extra Components}

In this section, we introduce some extra components that the STSGCN equiped to enhance its representation power.

Mask matrix For the graph convolutional operations in STSGCN, the adjacency matrix $A^{\prime}$ decides the weights of aggregation. However, each node has a different influence magnitude on its neighbors. If the adjacency matrix only contains 0 and 1 , the aggregation may be restricted. If the two nodes in the localized spatial-temporal graph are connected, even if they have no correlation at a certain period, their features will be aggregated. So we add a learnable mask matrix $W_{\text {mask }}$ in STSGCN to adjust the aggregation weights to make the aggregation more reasonable.

$W_{\text {mask }} \in \mathbb{R}^{3 N \times 3 N}$ denotes the mask matrix. We do the element-wise product between $W_{\text {mask }}$ and localized adjacency matrix $A^{\prime}$ to generate a weight adjusted localized adjacency matrix:

$$
A_{\text {adjusted }}^{\prime}=W_{\text {mask }} \otimes A^{\prime} \in \mathbb{R}^{3 N \times 3 N} .
$$

After that, we use $A_{\text {adjusted }}^{\prime}$ to compute all graph convolutions in our model.

Input layer We add a fully connected layer at the top of the network to transform the input into a high-dimension space, which can improve the representation power of the network.

Output layer We design an output layer to transform the output of the last STSGCL into the expected prediction. The input of this output layer can be denoted as $X \in \mathbb{R}^{T \times N \times C}$. We first transpose and reshape it to $X^{\top} \in \mathbb{R}^{N \times T C}$. Then we use $T^{\prime}$ two-fully-connected-layers to generate the prediction as follow:

$$
\hat{y}^{(i)}=\operatorname{Re} L U\left(X^{\top} W_{1}^{(i)}+b_{1}^{(i)}\right) \cdot W_{2}^{(i)}+b_{2}^{(i)},
$$

where $\hat{y}^{(i)}$ denotes the prediction in time step $i$. $W_{1}^{(i)} \in$ $\mathbb{R}^{T C \times C^{\prime}}, b_{1}^{(i)} \in \mathbb{R}^{C^{\prime}}, W_{2}^{(i)} \in \mathbb{R}^{C^{\prime} \times 1}, b_{2}^{(i)} \in \mathbb{R}$ are learnable 
Table 1: Dataset description.

\begin{tabular}{ccc}
\hline Datasets & Number of sensors & Time range \\
\hline PEMS03 & 358 & $9 / 1 / 2018-11 / 30 / 2018$ \\
PEMS04 & 307 & $1 / 1 / 2018-2 / 28 / 2018$ \\
PEMS07 & 883 & $5 / 1 / 2017-8 / 31 / 2017$ \\
PEMS08 & 170 & $7 / 1 / 2016-8 / 31 / 2016$ \\
\hline
\end{tabular}

parameters, $C^{\prime}$ denotes the number of features of the output of the first fully-connected layer. Then we concatenate all predictions of each time step into one matrix:

$$
\hat{Y}=\left[\hat{y}^{(1)}, \hat{y}^{(2)}, \ldots, \hat{y}^{(T)}\right] \in \mathbb{R}^{N \times T^{\prime}},
$$

where $\hat{Y}$ is the output of the overall STSGCN.

Loss function We select Huber loss (Huber 1992) as the loss function. The Huber loss is less sensitive to outliers than the squared error loss.

$$
L(Y, \hat{Y})= \begin{cases}\frac{1}{2}(Y-\hat{Y})^{2} & |Y-\hat{Y}| \leq \delta \\ \delta|Y-\hat{Y}|-\frac{1}{2} \delta^{2} & \text { otherwise }\end{cases}
$$

where $Y$ denotes the ground truth and $\hat{Y}$ denotes the prediction of the model, $\delta$ is a threshold parameter which controls the range of squared error loss.

\section{Experiments}

We evaluate the performance of STSGCN on four highway traffic datasets. These data are collected from the Caltrans Performance Measurement System (PeMS) (Chen et al. 2001).

\section{Datasets}

We construct four different datasets from 4 districts respectively, namely PEMS03, PEMS04, PEMS07 and PEMS08. The flow data is aggregated to 5 minutes, which means there are 12 points in the flow data for each hour. We use traffic flow data from the past hour to predict the flow for the next hour. The detailed information is shown in Table 1.

The spatial networks for each dataset is constructed according to the actual road network. If the two monitors are on the same road, the two points are considered to be connected in the spatial network.

We standardize the features by removing the mean and scaling to unit variance with:

$$
X^{\prime}=\frac{X-\operatorname{mean}(X)}{\operatorname{std}(X)}
$$

where mean $(X)$ and $\operatorname{std}(X)$ are the mean and the standard deviation of the historical time series, respectively.

\section{Baseline Methods}

- VAR (Hamilton 1994): Vector Auto-Regression is an advanced time series model, which can capture the pairwise relationships among time series.
- SVR (Drucker et al. 1997): Support Vector Regression uses a linear support vector machine for regression tasks.

- LSTM (Hochreiter and Schmidhuber 1997): Long ShortTerm Memory Network for time series prediction.

- DCRNN (Li et al. 2017): Diffusion Convolutional Recurrent Neural Network utilizes diffusion graph convolutional networks and seq2seq to encode spatial information and temporal information, respectively.

- STGCN (Yu, Yin, and Zhu 2018): Spatial-Temporal Graph Convolutional Network. STGCN uses ChebNet and 2D convolutional networks to capture spatial dependencies and temporal correlations, respectively.

- ASTGCN(r) (Guo et al. 2019a): Attention Based SpatialTemporal Graph Convolutional Networks designs spatial attention and temporal attention mechanisms to model spatial and temporal dynamics, respectively. ASTGCN integrates three different components to model periodicity of highway traffic data. In order to ensure the fairness of comparison experiments, we only take its recent components.

- STG2Seq (Bai et al. 2019): Spatial-Temporal Graph to Sequence Model uses multiple gated graph convolutional module and seq2seq architecture with attention mechanisms to make multi-step prediction.

- Graph WaveNet (Wu et al. 2019): Graph WaveNet combines graph convolution with dilated casual convolution to capture spatial-temporal dependencies.

\section{Experiment Settings}

We split all datasets with ratio $6: 2: 2$ into training sets, validation sets and test sets. We use one hour historical data to predict the next hour's data, which means using the past 12 continuous time steps to predict the future 12 continuous time steps. All experiments are repeated ten times.

We implement the STSGCN model using MXNet (Chen et al. 2015). The hyperparameters are determined by the model's performance on the validation datasets. The best model on these four datasets consists of 4 STSGCLs, each STSGCM contains 3 graph convolutional operations with 64, 64, 64 filters respectively.

\section{Experiment Results}

Table 2 shows the comparsion of different approaches for the forecasting tasks. Our STSGCN consistently outperforms other baseline methods on three datasets except for PEMS07. In PEMS07, our STSGCN has the best MAE and MAPE, except for the RMSE which is slightly larger than the that of DCRNN.

VAR, SVM and LSTM only take temporal correlations into consideration and cannot utilize the spatial dependencies of the spatial-temporal network. DCRNN, STGCN, ASTGCN(r), STG2Seq and our STSGCN all take advantages of spatial information, so they have better performance than the methods only for time series prediction.

DCRNN, STGCN, ASTGCN, and Graph WaveNet use two module to model spatial dependencies and temporal correlations respectively. And they share one module with all 
Table 2: Performance comparison of different approaches for traffic flow forecasting.

\begin{tabular}{|c|c|c|c|c|c|c|c|c|c|c|}
\hline \multicolumn{2}{|c|}{ Baseline methods } & \multirow{2}{*}{ VAR } & \multirow{2}{*}{ SVR } & \multirow{2}{*}{ LSTM } & \multirow{2}{*}{ DCRNN } & \multirow{2}{*}{ STGCN } & \multirow{2}{*}{ ASTGCN(r) } & \multirow{2}{*}{ STG2Seq } & \multirow{2}{*}{ Graph WaveNet } & \multirow{2}{*}{ STSGCN } \\
\hline Datasets & Metrics & & & & & & & & & \\
\hline \multirow{3}{*}{ PEMS03 } & MAE & 23.65 & $21.97 \pm 0.00$ & $21.33 \pm 0.24$ & $18.18 \pm 0.15$ & $17.49 \pm 0.46$ & $17.69 \pm 1.43$ & $19.03 \pm 0.51$ & $19.85 \pm 0.03$ & $\mathbf{1 7 . 4 8} \pm \mathbf{0 . 1 5}$ \\
\hline & MAPE (\%) & 24.51 & $21.51 \pm 0.46$ & $23.33 \pm 4.23$ & $18.91 \pm 0.82$ & $17.15 \pm 0.45$ & $19.40 \pm 2.24$ & $21.55 \pm 1.68$ & $19.31 \pm 0.49$ & $16.78 \pm 0.20$ \\
\hline & RMSE & 38.26 & $35.29 \pm 0.02$ & $35.11 \pm 0.50$ & $30.31 \pm 0.25$ & $30.12 \pm 0.70$ & $29.66 \pm 1.68$ & $29.73 \pm 0.52$ & $32.94 \pm 0.18$ & $29.21 \pm \mathbf{0 . 5 6}$ \\
\hline \multirow{3}{*}{ PEMS04 } & MAE & 23.75 & $28.70 \pm 0.01$ & $27.14 \pm 0.20$ & $24.70 \pm 0.22$ & $22.70 \pm 0.64$ & $22.93 \pm 1.29$ & $25.20 \pm 0.45$ & $25.45 \pm 0.03$ & $21.19 \pm 0.10$ \\
\hline & MAPE (\%) & 18.09 & $19.20 \pm 0.01$ & $18.20 \pm 0.40$ & $17.12 \pm 0.37$ & $14.59 \pm 0.21$ & $16.56 \pm 1.36$ & $18.77 \pm 0.85$ & $17.29 \pm 0.24$ & 13.90 \\
\hline & RMSE & 36.66 & $44.56 \pm 0.01$ & $41.59 \pm 0.21$ & $38.12 \pm 0.26$ & $35.55 \pm 0.75$ & $35.22 \pm 1.90$ & $38.48 \pm 0.50$ & $39.70 \pm 0.04$ & $33.65 \pm 0.20$ \\
\hline \multirow{3}{*}{ PEMS07 } & MAE & 75.63 & $32.49 \pm 0.00$ & $29.98 \pm 0.42$ & $25.30 \pm 0.52$ & $25.38 \pm 0.49$ & $28.05 \pm 2.34$ & $32.77 \pm 3.21$ & $26.85 \pm 0.05$ & $24.26 \pm 0.14$ \\
\hline & MAPE (\%) & 32.22 & $14.26 \pm 0.03$ & $13.20 \pm 0.53$ & $11.66 \pm 0.33$ & $11.08 \pm 0.18$ & $13.92 \pm 1.65$ & $20.16 \pm 4.36$ & $12.12 \pm 0.41$ & 10.21 \\
\hline & RMSE & 115.24 & $50.22 \pm 0.01$ & $45.84 \pm 0.57$ & $38.58 \pm 0.70$ & $38.78 \pm 0.58$ & $42.57 \pm 3.31$ & $47.16 \pm 3.66$ & $42.78 \pm 0.07$ & $39.03 \pm 0.27$ \\
\hline \multirow{3}{*}{ PEMS08 } & MAE & 23.46 & $23.25 \pm 0.01$ & $22.20 \pm 0.18$ & $17.86 \pm 0.03$ & $18.02 \pm 0.14$ & $18.61 \pm 0.40$ & $20.17 \pm 0.49$ & $19.13 \pm 0.08$ & $\mathbf{1 7 . 1 3} \pm \mathbf{0 . 0 9}$ \\
\hline & MAPE (\%) & 15.42 & $14.64 \pm 0.11$ & $14.20 \pm 0.59$ & $11.45 \pm 0.03$ & $11.40 \pm 0.10$ & $13.08 \pm 1.00$ & $17.32 \pm 1.14$ & $12.68 \pm 0.57$ & $\mathbf{1 0 . 9 6} \pm \mathbf{0 . 0 7}$ \\
\hline & RMSE & 36.33 & $36.16 \pm 0.02$ & $34.06 \pm 0.32$ & $27.83 \pm 0.05$ & $27.83 \pm 0.20$ & $28.16 \pm 0.48$ & $30.71 \pm 0.61$ & $31.05 \pm 0.07$ & $26.80 \pm 0.18$ \\
\hline
\end{tabular}

different periods to extract the long-range spatial-temporal correlations, which ignores the heterogeneities in spatialtemporal network data. Our method take localized spatialtemporal correlations into account and capture the heterogeneities in spatial-temporal data, so our STSGCN has better performance than these methods.

STG2Seq also intends to model the spatial-temporal correlations simultaneously. As we can see from the Table 2, our STSGCN has better performance on the four datasets. The limitation of STG2Seq is that it simply concatenates the features of the neighboring periods, rather than treating the nodes at different time steps as different individual nodes like our STSGCN. To some extent this approach ignores temporal information and spatial-temporal correlations.

\section{Component Analysis}

To further investigate the effect of different modules of STSGCN, we design six variants of the STSGCN model. We compare these six variants with the STSGCN model on the PEMS03 dataset. All of these models contains four STSGCLs with $[64,64,64]$ filters in each STSGCM. The difference of these seven models are described as below:

1. basic: This model does not equip with spatial-temporal embeddings and mask matrix. It uses ReLU as the activation function. Each STSGCL only contains one STSGCM, and it shares this module in all time periods. The output layer is the same as ASTGCN's output layer which simply uses a convolutional layer with 12 filters to generate predictions.

2. mulit-module: This model changes the STSGCLs in the basic model to multi-module version.
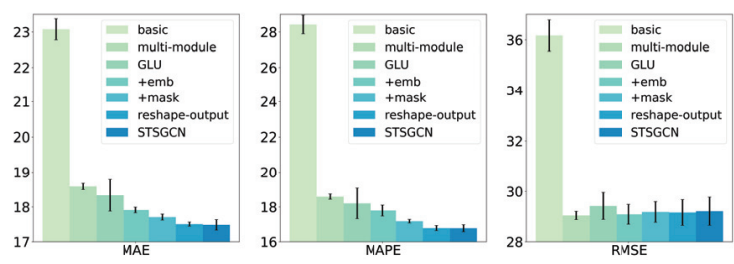

Figure 5: Component analysis of STSGCN.
3. GLU: This model changes all of activation function in multi-module model to GLU.

4. +emb: This model adds spatial-temporal embeddings in each STSGCL based on GLU version.

5. +mask: This model adds mask matrix based on +emb.

6. reshape-output: This model uses two fully connected layers to generate the expected predictions.

7. STSGCN: The STSGCN model deploys multiple twofully-connected layers to generate predictions of each time step.

As Figure 5 illustrates, the GLU have better performance than ReLU activation function. The reason for that is GLU has twice the parameter size of ReLU, so its larger capacity enables it to captures complex spatial-temporal correlations. Besides, it can control the output more flexibly than ReLU.

The model that equips with individual STSGCMs for each time period outperforms the shared STSGCM by a large margin, which shows the necessity of modeling the heterogeneities in spatial-temporal network data.

Besides, the results show that the spatial-temporal embedding can obviously improve the performance of the STSGCN model.

We add the mask matrix to tune the weights between each node and its neighbors in graph convolution operations. It can improve the forecasting performance a little.

The convolutional layer for generating the prediction does not make sense, because it shares all the parameters with all nodes in spatial graphs. Due to the heterogeneity in spatialtemporal data, each node may exhibit different properties, so using different modules to generate the prediction results is better than one convolutional output layer. So the reshaped output layer and multi-output version can further improve the prediction performance.

\section{Conclusion}

We propose a model which can not only capture the localized spatial-temporal correlations effectively but also take the heterogeneities in spatial-temporal data into considerations. And extensive experiments on four real-world datasets show that our model is superior to the existing models. Besides, our proposed model is a generalframework for spatial-temporal network data forecast- 
ing, so it can be applied in many related applications. The code and datasets have been released at: https://github.com/Davidham3/STSGCN.

\section{Acknowledgments}

This work was supported by the Fundamental Research Funds for the Central Universities (Grant No. 2019JBM024).

\section{References}

Bai, L.; Yao, L.; Kanhere, S.; Wang, X.; and Sheng, Q. 2019. Stg2seq: Spatial-temporal graph to sequence model for multi-step passenger demand forecasting. In IJCAI.

Bruna, J.; Zaremba, W.; Szlam, A.; and LeCun, Y. 2013. Spectral networks and locally connected networks on graphs. CoRR abs/1312.6203.

Chen, C.; Petty, K.; Skabardonis, A.; Varaiya, P.; and Jia, Z. 2001. Freeway performance measurement system: mining loop detector data. Transportation Research Record 1748(1):96-102.

Chen, T.; Li, M.; Li, Y.; Lin, M.; Wang, N.; Wang, M.; Xiao, T.; Xu, B.; Zhang, C.; and Zhang, Z. 2015. Mxnet: A flexible and efficient machine learning library for heterogeneous distributed systems. ArXiv abs/1512.01274.

Dauphin, Y. N.; Fan, A.; Auli, M.; and Grangier, D. 2017. Language modeling with gated convolutional networks. In Proceedings of the 34th International Conference on Machine Learning-Volume 70, 933-941. JMLR. org.

Defferrard, M.; Bresson, X.; and Vandergheynst, P. 2016. Convolutional neural networks on graphs with fast localized spectral filtering. In Advances in neural information processing systems, 3844-3852.

Drucker, H.; Burges, C. J.; Kaufman, L.; Smola, A. J.; and Vapnik, V. 1997. Support vector regression machines. In Advances in neural information processing systems, 155-161.

Gehring, J.; Auli, M.; Grangier, D.; Yarats, D.; and Dauphin, Y. N. 2017. Convolutional sequence to sequence learning. In Proceedings of the 34th International Conference on Machine Learning-Volume 70, 1243-1252. JMLR. org.

Graves, A. 2013. Generating sequences with recurrent neural networks. arXiv preprint arXiv:1308.0850.

Guo, S.; Lin, Y.; Feng, N.; Song, C.; and Wan, H. 2019a. Attention based spatial-temporal graph convolutional networks for traffic flow forecasting. In Proceedings of the AAAI Conference on Artificial Intelligence, volume 33, 922-929.

Guo, S.; Lin, Y.; Li, S.; Chen, Z.; and Wan, H. 2019b. Deep spatial-temporal 3d convolutional neural networks for traffic data forecasting. IEEE Transactions on Intelligent Transportation Systems.

Hamilton, W.; Ying, Z.; and Leskovec, J. 2017. Inductive representation learning on large graphs. In Advances in Neural Information Processing Systems, 1024-1034.

Hamilton, J. D. 1994. Time series analysis, volume 2. Princeton university press Princeton, NJ.
Hochreiter, S., and Schmidhuber, J. 1997. Long short-term memory. Neural computation 9(8):1735-1780.

Huber, P. J. 1992. Robust estimation of a location parameter. In Breakthroughs in statistics. Springer. 492-518.

Kipf, T. N., and Welling, M. 2017. Semi-supervised classification with graph convolutional networks. In International Conference on Learning Representations (ICLR).

Li, Y.; Yu, R.; Shahabi, C.; and Liu, Y. 2017. Diffusion convolutional recurrent neural network: Data-driven traffic forecasting. In ICLR.

Shi, X.; Chen, Z.; Wang, H.; Yeung, D.-Y.; Wong, W.-K.; and Woo, W.-c. 2015. Convolutional 1stm network: A machine learning approach for precipitation nowcasting. In $A d-$ vances in neural information processing systems, 802-810.

Veličković, P.; Cucurull, G.; Casanova, A.; Romero, A.; Liò, P.; and Bengio, Y. 2018. Graph Attention Networks. International Conference on Learning Representations. accepted as poster.

Williams, B. M., and Hoel, L. A. 2003. Modeling and forecasting vehicular traffic flow as a seasonal arima process: Theoretical basis and empirical results. Journal of transportation engineering 129(6):664-672.

Wu, Z.; Pan, S.; Long, G.; Jiang, J.; and Zhang, C. 2019. Graph wavenet for deep spatial-temporal graph modeling. In IJCAI.

Xu, K.; Li, C.; Tian, Y.; Sonobe, T.; ichi Kawarabayashi, K.; and Jegelka, S. 2018. Representation learning on graphs with jumping knowledge networks. In ICML.

Yu, B.; Yin, H.; and Zhu, Z. 2018. Spatio-temporal graph convolutional networks: A deep learning framework for traffic forecasting. In IJCAI.

Zhang, J.; Zheng, Y.; and Qi, D. 2017. Deep spatio-temporal residual networks for citywide crowd flows prediction. In Thirty-First AAAI Conference on Artificial Intelligence. 